\title{
Controlled Release of Antipyrine from Tablets Using Synthesized Copolymers as Matrices in Gastric Medium
}

\author{
Hanane Merine and Haouaria Merine* \\ Laboratory of Macromolecular Physical and Organic Chemistry, Faculty of Exact Sciences, DjilaliLiabes Universty, BP89 City El Arbi \\ Ben m' hidiSidi Bel-Abbes, ALGERIA.
}

\begin{abstract}
Introduction: In order to control and modify the drug release, several types of devices were used to obtain a controlled release of the organic drug. A drug delivery system can be a matrix of polymer incorporating the active agent. New solid dosage forms (tablets) composed from the active agent (Antipyrine) and copolymers synthesized: Poly (vinyl acetate-co-methyl methacrylate) and Poly (vinyl pyrrolidone-co-methyl methacrylate) able to control drug release have been prepared and investigated in this paper. Methods: These copolymers synthesized were characterized (FTIR, ${ }^{13} \mathrm{C} \mathrm{RMN}, \mathrm{Tg}$ and Mv), solid dosage forms (tablets): T(VAc1), T(Vac2) and T(VP) composed from the active agent (AP) and copolymers synthesized: poly (VAc-co-MMA) and poly (VP-co-MMA) as matrices were elaborated. The drug release from these formulations are performed in a cylindrical double-wall glass reactor $(100 \mathrm{~mL})$, kept at a temperature of $(37 \pm 0.5)^{\circ} \mathrm{C}$ and was followed using UV-Vis spectrophotometer in acidic medium at $\mathrm{pH} 1.2$. The effect of the matrix on the drug release was studied. Results: The drug dissolution from matrix tablets is significantly influenced by the nature of matrix. The results showed that after $2 \mathrm{~h}$ (the time corresponding to the drug retention in the human stomach), the percentages of the drug released from $\mathrm{T}(\mathrm{VAc} 1), \mathrm{T}(\mathrm{Vac} 2)$ and $\mathrm{T}(\mathrm{VP})$ are $1.04 \%, 0.37 \%$, and $36.89 \%$ respectively. The release is even greater when the matrix is hydrophilic. Swellable matrix tablets, such as poly (vinyl acetate-co-methyl methacrylate) tablets, are activated by water, and drug release is controlled by the interaction between water, polymer and drug. Theoretical analyses of the kinetics of controlled release of AP have been established and Antipyrine dissolution rate constants were calculated from Higuchi'srelease model and the $n$ exponents from Korsmeyer-Peppas model were determinate. Conclusion: The suitable kinetics model for describing the release of AP from the matrix tablets was the Higuchi model. The coefficients of correlation $r^{2}$ in this model are above 0.99. The results of the Korsmeyer-Peppas equation, the values of $n$ are in agreement with the experimental results already reported.
\end{abstract}

Key words: Antipirine, Drug release, Poly (vinylacetate-co-methyl methacrylate), Poly(vinylpyrrolidone-co-methylmethacrylate), Tablets.

\section{INTRODUCTION}

In order to increase the acceptability or efficacy of oral drug therapy, many drugs are presented as controlled release formulation. ${ }^{1}$ Several types of devices ${ }^{2,3}$ were used to obtain a controlled release of the organic drug: a drug delivery system may be a matrix of polymer incorporating the organic sub- stance. However, this technique was also employed for preparing sustained-release forms of various water-soluble and shortacting drugs using water-insoluble inert carriers. ${ }^{4,5}$ Examples of such water-insoluble carriers are Eudragits, a group of commercially available acrylate and methacrylate
Submission Date :22-01-0215 Revision Date : :18-02-2015 Accepted Date : :02-04-2015

DOI: $10.5530 /$ ijper.49.4.7 Correspondence Address Dr. HaouariaMerine Faculty of Exact Sciences, DjilaliLiabesUniversty, BP89 City El ArbiBen m' hidiSidi Bel-Abbes, ALGERIA Email:merine_houaria@ yahoo.fr

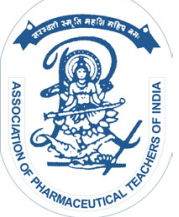

www.ijper.org 


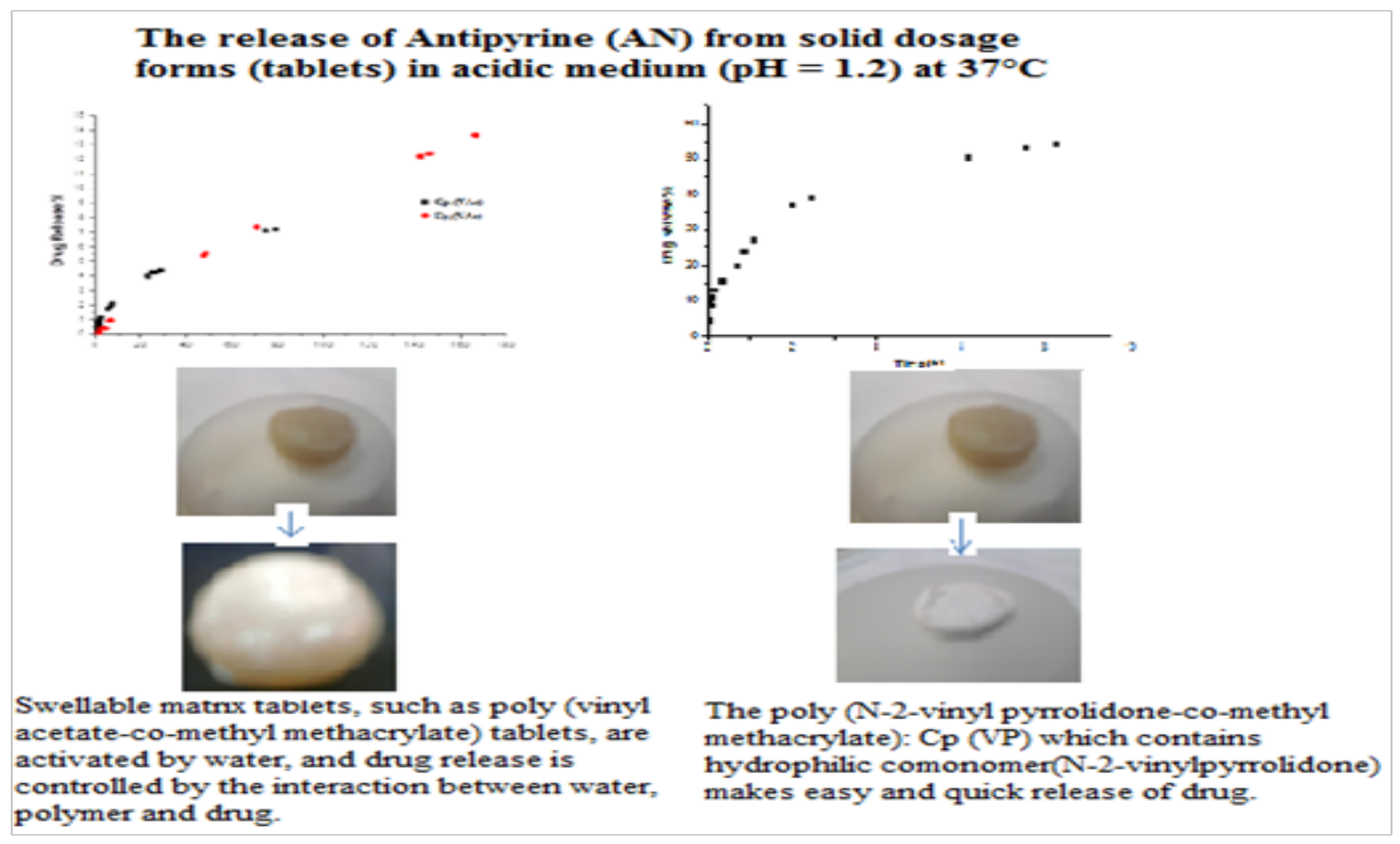

\section{Graphical Abstract}

polymers and copolymers available in anionic, cationic, and zwitterionic forms ${ }^{6,7}$ Hydroxypropyl methylcellulose HPMC, ${ }^{8}$ Ethyl cellulose, ${ }^{9}$ and poly(methylmethacrylateco-methacrylic acid). ${ }^{10}$ Hence, traditional dosage forms such as tablets still offer important advantages if they are able to decrease the total daily dosage of a medicinal agent. ${ }^{11}$ These systems are based on a wide range of biopolymers. ${ }^{12-14}$. Recently, Diaf et al ${ }^{15}$ have prepared polymeric tablets based on piroxicam. These Tablets are compounds of ethylcellulose, Eudragit or mixtures of Eudragit and synthesized poly (oxepan-2-one): (poly(caprolactone); Pcl. The release of active substance is then often controlled by diffusion through the matrix and/or swelling and/or erosion of the latter.

In the present article, our experiences in the use of copolymers based on methylmethacrylate for oral con- trolled drug delivery are highlighted. The objective of this study was to prepare and evaluate solid dosage forms (discs) for the controlled-release of antipyrine (AP) Antipyrine (Phenazone) is strongly resorbed by the human blood by digestive way. It is an analgesic and both antipyretic and anti-inflammatory agent. ${ }^{16}$

In our first part, the methylmethacrylate was copolymerized with vinyl acetate on the one hand and with $\mathrm{N}$-2-vinylpyrrolidone secondly by a radical copolymerization. In the second part, solid dosage forms (tablets) composed from the active agent (AP) and copolymers synthesized: poly (VAc-co-MMA) and poly (VP-coMMA) as matrices were elaborated in order to modify the drug release. In these experiments, the effect of matrix on the drug release was studied.

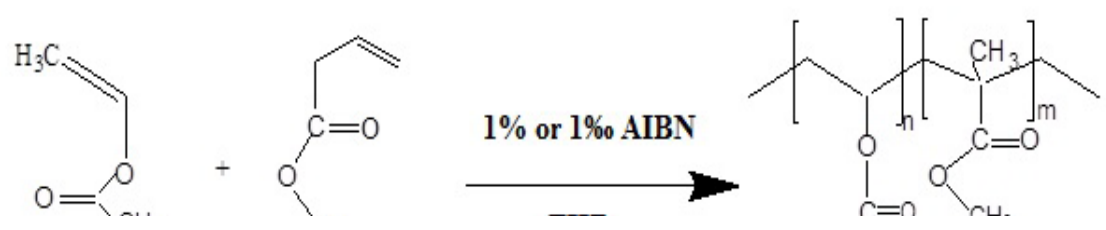

Scheme 1: Chemical reaction of $C p_{1}$ (VAc) and $C p_{2}$ (VAc) 


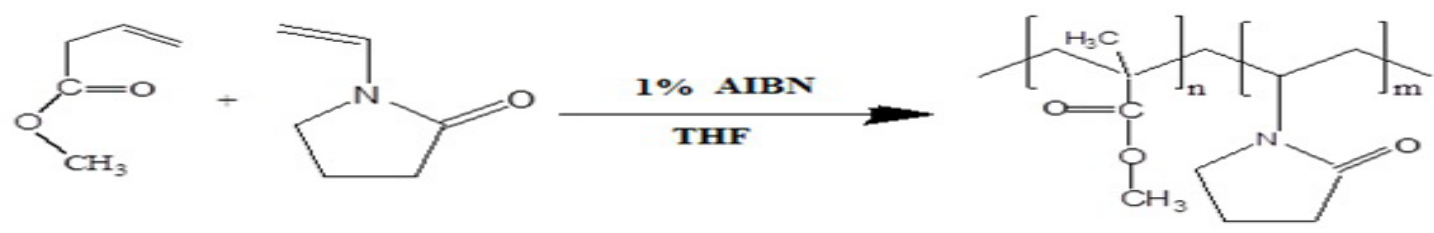

Scheme 2: Chemical reaction of Cp (VP)

\begin{tabular}{|c|c|c|c|c|c|}
\hline \multicolumn{6}{|c|}{ Table 1: Experimental conditions of the synthesis of copolymers } \\
\hline Copolymer & Monomer & Comonomer & $\%$ AIBN & Reaction time & Yield \\
\hline Cp $_{1}$ (VAc) & MMA & VAc & $1 \%$ & $33 \mathrm{~h}$ & $85 \%$ \\
\hline $\mathbf{C p}_{2}$ (VAc) & MMA & VAc & $1 \%$ & $48 \mathrm{~h}$ & $81 \%$ \\
\hline $\mathbf{C p}$ (VP) & MMA & VP & $1 \%$ & $3 \mathrm{~h} 39 \mathrm{mn}$ & $73 \%$ \\
\hline
\end{tabular}

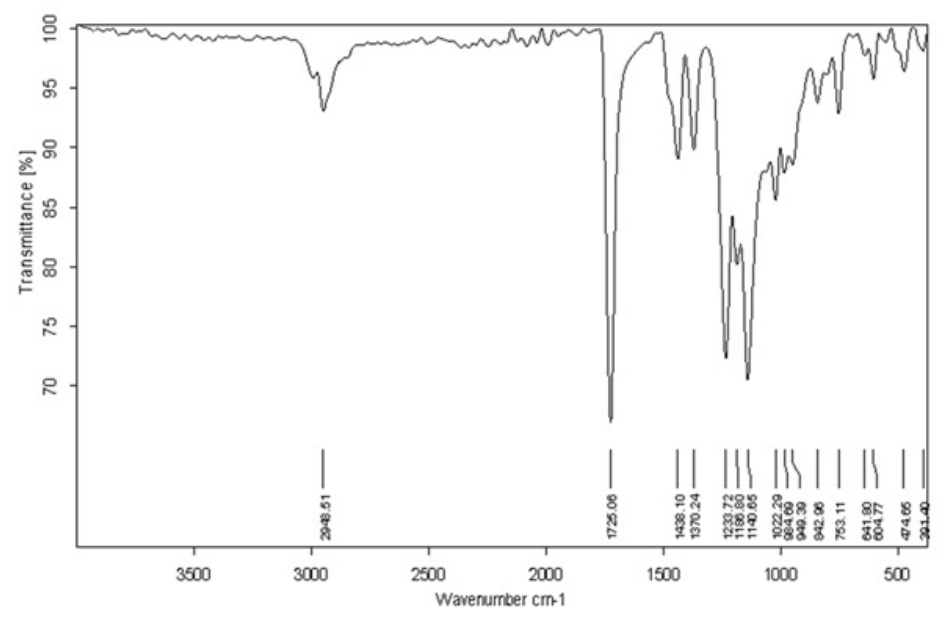

Figure 1: Infrared spectrum of Cp1(VAc)

The kinetics of (AP) released from tablets have been measured by using a double-bean UVspectrophotometer at synthetic gastric liquid $(\mathrm{pH}=1,2)$ under the same conditions of temperature $\left(37^{\circ} \mathrm{C}\right)$ and stirring $(500 \mathrm{rpm})$.

Finally, several mathematical models recording to the First order, the Higuchi's, the Korsmeyer-Peppar's and Hixson-Crowell's equations, have been developed, for elucidate the drug transport processes and to predict the resulting drug release kinetics. ${ }^{17-21}$

\section{Experimental part}

\section{Synthesis of Copolymers}

The copolymers $\left(\mathrm{Cp}_{1}(\mathrm{VAc}), \mathrm{Cp}_{2}(\mathrm{VAc})\right.$ and $\mathrm{Cp}(\mathrm{VP})$ were obtained by a radical copolymerization, under nitrogen atmosphere, in anhydrous tetrahydrofuran (THF) as solvent, at $65^{\circ} \mathrm{C}$, and in the presence of two percentage of initiator: $1 \%$ or $0,1 \%$ of AIBN azobisisobutyronitrile as the case during variable reactional times.
In a glass tube, the same quantities in $\mathrm{mol}(25 \mathrm{mmol})$ of methylmethacrylate (MMA) and vinylacetate (VAc) or Vinylpyrrolidone (VP) were dissolved in $5 \mathrm{~mL}$ of THF with the presence of AIBN as initiator $1 \%$ or $0.1 \%$ as the case) Scheme 1 and 2. After degassing, the glass tube is immersed in thermostat oil bath at $65^{\circ} \mathrm{C}$. The tube was removed once the solution became viscous. This one is solubilized with a minimum amount of THF to take it less viscous. The purification of our copolymers has established by fractional precipitation method by addition of non-solvent. ${ }^{22}$ After several operation of "solubilizing-precipitation", the solid copolymers (white powder) are separated by vacuum filtration. Table 1 summarizes the experimental conditions of the synthesis of copolymers.

\section{Characterization of the copolymers}

\section{Infrared analysis of the copolymers}

The Infrared spectra were recorded on a Bruker Alpha FT-IR spectrometer (equipped with Alpha's Platinum 
Table 2: Values of ${ }^{13} \mathrm{C}$ NMR signals of copolymers

\begin{tabular}{|c|c|c|}
\hline- & $\mathbf{C p}$ (VAc) & $\mathbf{C p}$ (VP) \\
\hline $\mathrm{C}=\mathrm{O}$ & 170,33 & 175,42 \\
\hline $\mathrm{O}-\underline{\mathrm{CH}}_{3}$ & 51,77 & 51,74 \\
\hline$\underline{\mathrm{C}}_{2}-{ }^{-}$ & $31,85-44,54$ & $31,23-44,83$ \\
\hline $\mathrm{C}-\underline{\mathrm{H}}_{3}$ & 20,97 & 18,28 \\
\hline
\end{tabular}

Table 3:Tg values of copolymers

\begin{tabular}{|c|c|c|c|}
\hline Copolymers & $\mathbf{C p} 1$ (VAc) & $\mathbf{C p} 2$ (VAc) & $\mathbf{C p}$ (VP) \\
\hline $\mathbf{T g}\left({ }^{\circ} \mathbf{C}\right)$ & 77,9 & 98,3 & 120,9 \\
\hline
\end{tabular}

Table 4: Viscometric masses of the copolymers

\begin{tabular}{|c|c|}
\hline Copolymers & Mvg/Mol \\
\hline Cp1(VAc) & 6723 \\
\hline Cp2(VAc) & 9378 \\
\hline Cp(VP) & 20797 \\
\hline
\end{tabular}

ATR single reflection diamond ATR module), the principal IR bands are $\sigma\left(\mathrm{cm}^{-1}\right)$ :

$\mathrm{Cp}_{1}$ (VAc) and $\mathrm{Cp}_{2}$ (VAc): 1725,06: C=O (ester); 1140,65: $\mathrm{C}-\mathrm{O}$ (ester); 2948,51: -C-H, stretch; 1438,10: $-\mathrm{CH}_{3}$ (bending) Figure 1.

Cp(VP):1654,69: $\quad \mathrm{C}=\mathrm{O}(\mathrm{VP}) ; \quad$ 1722,82: $\mathrm{C}=\mathrm{O}($ ester); 1189,89: (C-O); 2950,23: C-H (alcane, stretch); 1428,23: $-\mathrm{CH}_{3}$, (bending).

\section{Nuclear Magnetic Resonance ${ }^{13} \mathrm{C}$ NMR}

The carbon magnetic resonance $\left({ }^{13} \mathrm{C}\right.$ NMR) spectra of copolymers were recorded using BRUCKER at $300 \mathrm{MHz}$.

The ${ }^{13} \mathrm{C}-\mathrm{NMR}$ spectra of copolymers give the $\delta$ (ppm) values resumed in table 2 . The carbonyl carbon $(>\mathrm{C}=\mathrm{O})$ signals of VP, VAc and MMA units appear between $\delta$ 170.33 and 175.42. The spectral region around $\delta 18,28-$ 51,74 is assigned to aliphatic carbon resonance in the back and side chain of VAc/MMA and VP/MMA copolymers. These values are in agreement with previous work. ${ }^{23}$

\section{Differential scanning Calorimetric DSC}

Analyzes were performed on a DSC device type DSC 204 F1 Phoenix- NETZSCH-GERATEBAU GMBH. The glass transition temperatures of the copolymers are given in Table 3.

\section{Viscometric analysis}

Viscometric measurements were performed using a capillary viscometer types KPG Cannon-Fenske. The temperature is maintained constant by means of a thermostatic $(25 \pm 0.1)^{\circ} \mathrm{C}$ bath and the average mass my of the copolymers were determined by viscometric measurements by applying the Mark-Houwink law. The measurement results are given in Table 4 .

\section{Tablet preparation}

The release kinetics of Antipyrine (AP) dispersed in the synthesized copolymers forming a solid dosage forms (discs) mass composition 80/20 were followed by UV-Vis spectrophotometer (Shimadzu UV-2401) in the physiological medium artificially reconstituted $\mathrm{pH}$ $=1.2$ at $37^{\circ} \mathrm{C} \cdot{ }^{24} \mathrm{~A}$ mixture of $80 \%$ copolymer and $20 \%$ (AP) is intimately dispersed and mixed in a dry mortar. It should be well crush the two powders for better homogenization. Of absolute ethanol sprays are then performed to obtain a thick mass easily malleable. For shaping the discs, the dough is placed in a mold carrier of a hydraulic press and the discs are formed after application of a low pressure sufficient to shape the disc. The dosage forms obtained are dried in a desiccator for several days to constant weight. The diameter (d) and thickness (h) of the discs were measured using a caliper. The values are averages of ten measurements. The characteristics of formulations are summarized in Table 5.

\section{RESULTS AND DISCUSSION}

\section{Drug release study}

The release kinetic studies of the active agent from discs are performed in a cylindrical double-wall glass reactor $(100 \mathrm{~mL})$, kept at a temperature of $(37 \pm 0.5){ }^{\circ} \mathrm{C}$ (temperature of the human body). The discs, inserted in a permeable fiber glass basket, were immersed in $100 \mathrm{~mL}$ of simulated liquid at $\mathrm{pH} 1,2$. The dissolution medium was stirred magnetically at a rotation speed of 500 $\mathrm{min}^{-1}$ to achieve good homogenization. Samples $(1 \mathrm{~mL})$ of the solution were collected for analysis at different time intervals. After an appropriate dilution, the sample was analyzed using a UV-VIS spectrophotometer at $\lambda_{\max }=230 \mathrm{~nm}$ of Antipyrine. The value of molar extinc-

\begin{tabular}{|c|c|c|c|c|c|}
\hline Table 5: Characteristics of dosage forms Cp / AP: $80 / 20$ \\
\hline Tablets & composition & $\mathbf{m}_{\mathbf{0}}(\mathbf{m g})$ & $\mathbf{m}_{\mathbf{i}}(\mathbf{m g})$ & $\mathbf{h}$ & $\mathbf{d}$ \\
\hline $\mathbf{T}$ (VAc1) & $\mathrm{Cp}_{1}$ (VAc)/AP & 397,2 & 79,44 & 0,510 & 1,05 \\
\hline $\mathbf{T}$ (VAc2) & $\mathrm{Cp}_{2}$ (VAc)/AP & 408,1 & 81,62 & 0,450 & 1,21 \\
\hline T (VP) & $\mathrm{Cp}_{3}$ (VP)/AP & 365 & 73 & 0,342 & 1,1066 \\
\hline
\end{tabular}
d-disc diameter, $\mathrm{h}$ - disc width, $\mathrm{m}_{\mathrm{o}}$ - disc mass, $\mathrm{m}_{\mathrm{i}}$ - initial mass of Antipirine in disc.


tion coefficient $\varepsilon_{\text {max }}\left(1 \cdot \mathrm{mole}^{-1} \cdot \mathrm{cm}^{-1}\right)=13031$ is calculated from the slope of the plot of $\mathrm{Abs}=\mathrm{f}(\mathrm{C})$ at $\mathrm{pH}=1.2$.

The release studies were carried out in a buffered solution at $\mathrm{pH} 1.2$ and $37^{\circ} \mathrm{C}$ and the influence of the matrix on the AP release was studied. All formulations were seen to be intact throughout the dissolution studies. The percentage of drug released from discs relative to time is shown in Figure 2 and Figure 3.

The effect of the matrix is noticeable, the results demonstrated that the release of Antipirine from the copolymer $\mathrm{Cp}_{3}(\mathrm{VP})$ is faster than the copolymers $\mathrm{Cp}_{1}(\mathrm{VAc})$ and $\mathrm{Cp}_{2}(\mathrm{VAc})$ in acidic medium. As a matter of fact, after $2 \mathrm{~h}$ (the time corresponding to the drug retention in the human stomach), the percentages of the drug released from $\mathrm{T}(\mathrm{VAc} 1)$, $\mathrm{T}(\mathrm{Vac} 2)$ and $\mathrm{T}(\mathrm{VP})$ are $1.04 \%$, $0.37 \%$, and $36.89 \%$ respectively. The amount of matter released depends upon its matrix.

The poly (N-2-vinylpyrrolidone-co-methylmethacrylate): $\mathrm{Cp}(\mathrm{VP})$ which contains hydrophilic comonomer (N-2-vinylpyrrolidone) makes easy and quick release of drug. In fact, the absorption rate of release medium by this tablet which facilitates dissolution of the drug and, subsequently, its diffusion.

As regards the $\mathrm{Cp}$ (VAc) matrix, the rate of the $\mathrm{AP}$ release is very slow. In effect, after 10h, compared with the last matrix, the percentage of drug released from $\mathrm{T}$ (VAc1) and $\mathrm{T}$ (VAc2) does not exceed $2.5 \%$ and $1.3 \%$ respectively which is clearly due to the low absorption of water by a tablet. These percentages of active agent are due to the amount of water that has penetrated after hydrolysis of vinyl acetate segments of copolymers $\mathrm{Cp} 1$ (VAc) and Cp2 (VAc). Figure 4 shows photographs that show the behavior of tablets T(VAc1), T(VAc2) and $\mathrm{T}(\mathrm{VP})$ before and after release kinetics of Antipyrine.

Copolymer (poly (vinylacetate-co-methylmethacrylate) $1 \%$ and $0.1 \%$ ) has an identity crisis. In effect, the alcohol

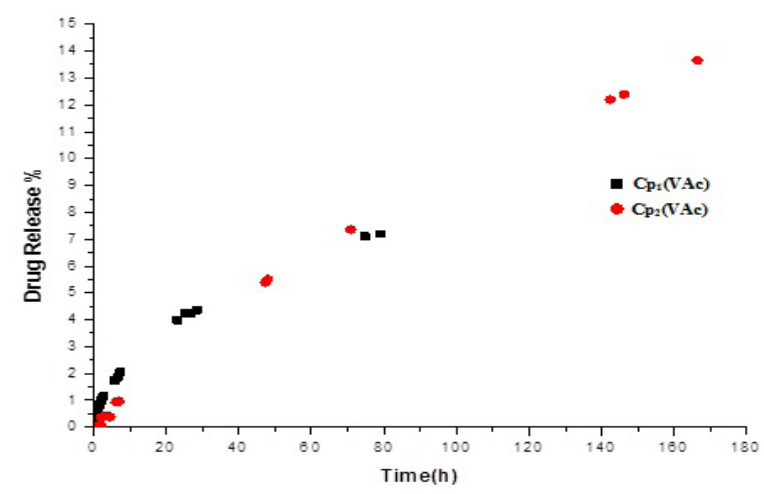

Figure 2: Release profiles of Antipirine from dosage forms (Cp1(VAc) / AP) and (Cp2 (VAc) / AP) at pH = 1,2 group obtained after hydrolysis of the acetate rush out to meet the water, but the acetate and MMA groups are hidden away in the middle of the copolymer (Scheme 3) and therefore to prevent the AP releases and to the outer surface like that of the dosage form become mushy as shown in Figure 4.

Swellable matrix tablets, such as poly(vinylacetate-comethylmethacrylate) tablets, are activated by water, and drug release is controlled by the interaction between water, polymer and drug. Hydration of polymer results in the formation of a gel layer that controls the drug release rate. ${ }^{25}$ When the penetration of water in the gel-matrix exceeds a critical concentration (i.e. the concentration at which the interactions between water and polymer increase, with a consequent reduction of polymer-polymer interactions), the polymer chains begin to separate, extending the spaces where distribution of the drug occurs (14\% of drug release after 170 hours).

\section{Release mechanisms and mathematical analysis}

The mathematical description of drug diffusion is often based on Fick's second law. ${ }^{26}$ In the literature, many theoritical or empirical models are described to explain this process of mass transfer. In our study, we chose four of them for quantitative prediction of controlled drug delivery. The selected models are: the first order, the Higuchi's ${ }^{17}$ the Korsmeyer's models ${ }^{18}$ and the HixsonCrowell's. ${ }^{19}$ Thus the choice of the best model is based on the value of $\mathrm{r}^{2}$, correlation coefficient obtained after tracing experimental results according to the equations corresponding to the selected models.

- First Order

$$
\log Q_{t}=\log Q_{o}+\frac{K_{1} \cdot t}{2,303}
$$

- Higuchi

$$
Q_{t}=K_{H} \sqrt{t}
$$

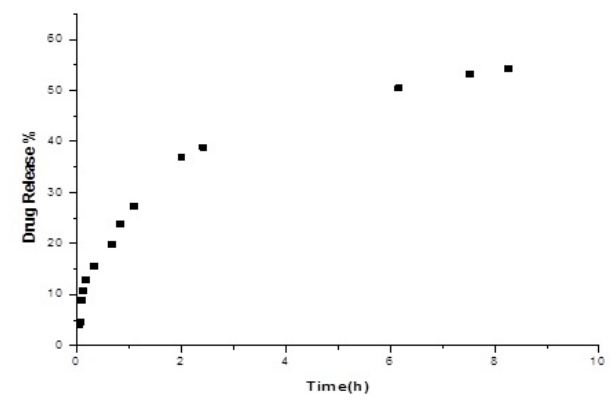

Figure 3: Release profile of Antipirine from dosage form $(\mathrm{Cp}(\mathrm{VP}) / \mathrm{AP})$ at $\mathrm{pH}=1,2$ 


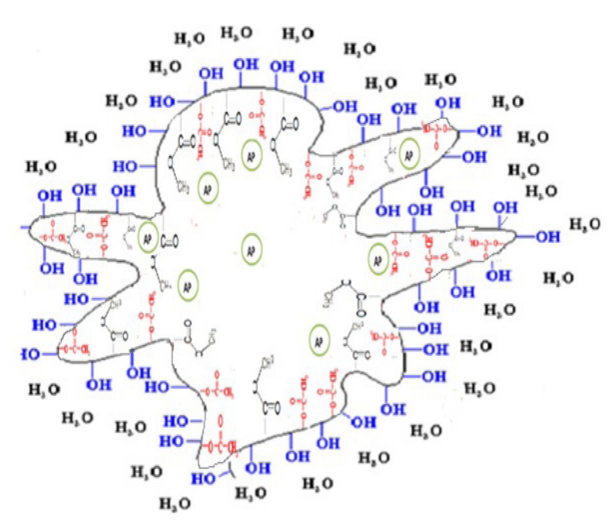

Scheme 3: Structure of copolymer (poly (vinylacetate-comethylmethacrylate) after hydrolysisof the acetate group

- Korsmeyer-Peppas

- Hixson-Crowell

$$
\frac{M_{t}}{M_{\infty}}=K_{K} t^{n}
$$

$$
\sqrt[3]{Q_{t}}-\sqrt[3]{Q_{r}}=K_{s} \cdot t
$$

Where $\mathrm{Q}_{\mathrm{t}}$ is the amount of drug dissolved in time $\mathrm{t} ; \mathrm{Q}_{0}$ is the initial amount of drug in the solution (most times $\mathrm{Q}_{0}=0$ ); Qi is the initial amount of drug in the pharmaceutical dosage form; $\mathrm{Qr}$ is the amount of drug remaining as a solid state at time $\mathrm{t} ; \mathrm{Mt} / \mathrm{M} \infty$ isthe fraction of drug released; $\mathrm{K}_{1}, \mathrm{~K}_{\mathrm{H}}, \mathrm{K}_{\mathrm{S}}$ and $\mathrm{K}_{\mathrm{K}}$ are, respectively the first order, the Higuchi's, the Hixson-Crowell's and the

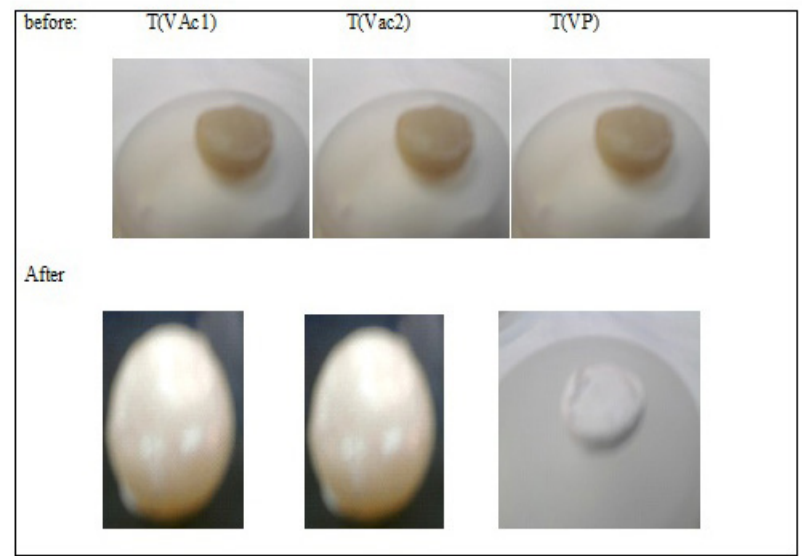

Figure 4: Photos representing the behavior of matrices before and after release of the AP in the physiological medium

Korsmeyer's release constants; and $n$ the release exponent that indicates of the mechanism of release. ${ }^{17-21}$

The Hixson-Crowell model assumes that the drug release is limited by the dissolution rate of the particles, and not by diffusion through the polymer matrix, ${ }^{20}$ and the Higuchi model able to describe the release of soluble or poorly soluble drug in water from a semi-solid or solid matrix system, ${ }^{17}$ Therefore, the value of $\mathrm{n}$ was used by Peppas ${ }^{26}$ in order to characterize the various release mechanisms. When $\mathrm{n}=0.5$ the release mechanism as Fick diffusion, and as a non-Fickian model if $\mathrm{n}$ is between 0.5 and 1.0 or $n=1.0$. When $n=0.5$, the drug release is controlled by diffusion and is time-dependent

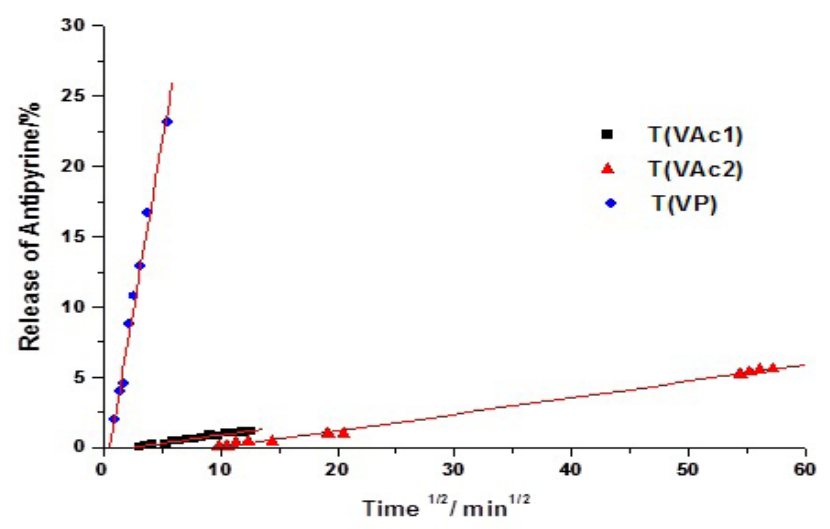

Figure 5: Fractional release of Antipyrine from Tablets as a function of the square root of time

Table 6: Co-efficients of correlation and dissolution rate constants of Antipyrine from tablets

\begin{tabular}{|c|c|c|c|c|c|c|c|c|c|}
\hline \multirow{2}{*}{ Formulation } & \multicolumn{2}{|c|}{ First Order } & \multicolumn{2}{|c|}{ Higuchi } & \multicolumn{2}{c|}{ Hixson-Crowell } & \multicolumn{3}{|c|}{ Korsmeyer-Peppa } \\
\cline { 2 - 12 } & $\mathbf{r}^{\mathbf{2}}$ & $\mathbf{K}_{\mathbf{1}}$ & $\mathbf{r}^{\mathbf{2}}$ & $\mathbf{K}_{\mathbf{H}}$ & $\mathbf{r}^{\mathbf{2}}$ & $\mathbf{K}_{\mathbf{s}}$ & $\mathbf{r}^{\mathbf{2}}$ & LnKK & $\mathbf{n}$ \\
\hline $\mathbf{T}(\mathbf{V A c 1})$ & 0,832 & 0,007 & 0,992 & 0,118 & 0,894 & 0,004 & 0,968 & $-9,4435$ & 1,03 \\
\hline $\mathbf{T}$ (VAc2) & 0,892 & $4,4.10^{-4}$ & 0,998 & 0,118 & 0,973 & $3,8.10^{-4}$ & 0,958 & $-11,416$ & 1,07 \\
\hline $\mathbf{T}(\mathbf{V P})$ & 0,822 & 0,031 & 0,996 & 4,851 & 0,879 & 0,049 & 0,987 & $-3,7906$ & 0,735 \\
\hline
\end{tabular}


while when $n=1.0$, the drug release is controlled by swelling and is time-independent with zero order kinetics. Values of $\mathrm{n}$ between 0.5 and 1.0 indicate superposition of both phenomena, known as anomalous transport. It is necessary to consider that the exponent values are valid for certain slab geometry, and different values can be derived for spheres and cylinders. ${ }^{27}$ The model which gave the highest coefficient of correlation $\mathrm{r}^{2}$ was considered to be the most suitable kinetic model for describing the release of AP from the matrix tablets.

Figure 5 gives plots of the fractional drug release as a function of the square root of time, and table 6 shows the coefficients of correlation and dissolution rate constants of Antipyrine from tablets according to the models studied. We have noted that the fractional release of Antipyrine is proportional to the square root of time during the short time as shown in Figure 5.

The suitable kinetics model for describing the release of AP from the matrix tablets was the Higuchi model. The coefficients of correlation $\mathrm{r}^{2}$ in this model are above 0.99. From the results of Higuchi's equation plots, the dissolution rate constants of drug varied from $0.118 \mathrm{~min}^{-}$ ${ }^{1 / 2}$ for $\mathrm{T}$ (VAc1) and $\mathrm{T}(\mathrm{VAc} 2)$ to $4.851 \mathrm{~min}^{-1 / 2}$ for $\mathrm{T}(\mathrm{VP})$. The effect of matrix on the dissolution rate is notable. When the matrix contains comonomer (N-2-vinylpyrrolidone), the drug release rate increases (4.851 $\mathrm{min}^{-1 / 2}$ ) and it is lower in the other matrix $\left(0.118 \mathrm{~min}^{-1 / 2}\right)$.

From the results of the Korsmeyer-Peppas equation, the values of $\mathrm{n}$ are different between tablets $\mathrm{T}(\mathrm{VAc} 1)$ and $T$ (VAc2) ( $n=1.03$ for $T$ (VAc1) and $n=1,07$ for $\mathrm{T}(\mathrm{VAc} 2))$ on one hand and tablet $\mathrm{T}(\mathrm{VP})(\mathrm{n}=0.735)$ on the other hand. These values are in agreement with the experimental results already reported. However, the mechanism of drug release from Tablets $T(V A c 1)$ and $\mathrm{T}(\mathrm{VAc} 2)$ is controlled by swelling, and from $\mathrm{T}(\mathrm{VP})$ the value of $\mathrm{n}$ is between $\mathrm{n}=0.5$ and $\mathrm{n}=1.0$, the mechanism of release is not well-known (superposition of both phenomena). It can be concluded that in vitro drug dis- solution from matrix tablets is significantly influenced by the nature of matrix.

\section{CONCLUSION}

This paper has paved the way to new oral forms able to control the release of drug in stomach. For it the copolymers Cp (VAc1), Cp (VAc2) and CP (VP) were synthesized and characterized. Various rates of drug release from tablets of different matrices were determined experimentally. The drug dissolution from matrix tablets is significantly influenced by the nature of matrix. Indeed the kinetics of drug release obtained by experiment and theory are in good agreement, proving the validity of the chosen model. Diffusion, swelling and dissolution are the governing mechanisms involved in the overall drug release process. Finally, the practical advantage of the models is to identify the mechanism of drug release from tablets of different matrices to achieve desired release profiles, thus facilitating the development of a new product controlled drug delivery i.e using other techniques principally microencapsulation in order to get a large domain of drug release.

\section{CONFLICT OF INTEREST}

None of the authors has a conflict of interest to disclose.

\section{ACKNOWLEDGEMENTS}

The authors present their thanks to Prof. A. MESLI, Director of the Laboratory of Macromolecular Physical and Organic Chemistry for his support and Pr K. Guemra, responsible of $\mathrm{PhD} 3^{\text {rd }}$ cycle, option: Organic Chemistry and Macromolecular for making available necessary facilities to complete this work. Also her thanks goes to Director of Polytechnic Military School (Bordj el Bahri) (Algiers); who assured for us; with a scientific spirit the characterization of the copolymers.

\section{Highlights of Paper}

- Themethylmethacrylate was copolymerized with vinyl acetate and with N-2-vinylpyrrolidone by a radical copolymerization.

- Solid dosage forms (tablets) composed from the active agent Antipyrine and copolymers synthesized: poly (VAc-co$\mathrm{MMA}$ ) and poly (VP-co-MMA) as matrices were elaborated in order to modify the drug release.

- The kinetics of (AP) released from tablets have been measured by using a double-bean UVspectrophotometer at synthetic gastric liquid $(\mathrm{pH}=1,2)$ under the same conditions of temperature $\left(37^{\circ} \mathrm{C}\right)$ and stirring $(500 \mathrm{rpm})$.

- The results showed that the release is even greater when the matrix is hydrophilic. The drug diffusivity is strongly dependent on the matrix swelling.

- Theoretical analyses of the kinetics of controlled release of AP have been established and Antipyrine dissolution rate constants were calculated from Higuchi'srelease model. 


\section{Author Profile}

- Dr MERINE Haouaria: $\mathrm{PhD}$ in Chemistry, presently working as Research Master in the Department Of Chemistry, Faculty of Exact Sciences, DjilaliLiabesUniversty of Sidi Bel Abbes (Algeria). She has 8 nos of journal paper.

- MERINE Hanane: Presently, she is a PhD student in 3rd year PhD 3rd cycle.

\section{REFERENCES}

1. Heilman K. Therapeutic systems-Rate-Controlled Drug Delivery, Concept and Development. Thieme Strallon. New York; 1984.

2. Florence AT. New drug delivery systems. Chem. Ind. 1993.

3. D'Antone S, Solaro R, Cheillini E, Rehab A, Akelah A, Issa R. Controlled release of herbicides loaded on oligoethylenoxylated styrene/divinylbenzene resins. New Polymeric Mater 1992; 3(4): 223-236.

4. Abd El Fettah S, Salib NN, El Assik M. A new approach for controlling the release rate of pheniramine aminosalicylate via solid dispersion in different types of Eudragit. Drug. Dev. Ind. Pharm.1984; 10(4): 649-66.

5. Hasegawa A, Nakagawa $H$, Sugimoto I. Bioavailability and stability of Nifedipine-enteric coating agent solid dispersion. Chem. Pharm. Bull. 1985; 33(1): 388-91.

6. Trapani A, Laquintana V, Denora N, Lopedota A, Cutrignelli A, Franco M, et al. Eudragit RS 100 microparticles containing 2-hydroxypropyl-betacyclodextrin and glutathione: physicochemical characterization, drug release and transport studies. Eur. J. Pharm. Sci. 2007; 30(1): 64-74.

7. Merine $\mathrm{H}$, Mesli A, Chafi $\mathrm{N}$. Study release in homogeneous and heterogeneous media of 3-phenylpropylamine grafted in copolymer support based on N-vinyl-2-pyrrolidone: Comparative study of the delayeffect, J. Mater. Environ. Sci. 2014; 5(5): 1390-7.

8. Shoaib MH, Tazeen J, Merchant, HA, Yousuf RI. Evaluation of drug release kinetics from ibuprofen matrix tablets using HPMC. Pak. J. Pharm. Sci. 2006; 19(2): 119-24.

9. El Bahri Z, Diaf K, Traveldet J-L, Chafi N. Drug release study of an herbicide from polymeric microspheres and disks. calcul of diffusivities. Phys. Chem. News. 2010; 53: 108-12.

10. Huang $\mathrm{J}$, Kao $\mathrm{H}$, Wu $\mathrm{XY}$. The $\mathrm{pH}$-dependent biphasic release of azidothymidine from a layered composite of PVA disks and P(MMA/MAA) spheres Journal of Controlled release 2000; 67(1): 45-54.

11. Sastry SV, Nyshadham JR, Fix JA. Recent technological advances in oral drugdelivery-a review. Pharmaceutical Science \& Technology Today 2000; 3(4): 138-45.

12. Prestwich GD, Luo Y. Novel biomaterials for drug delivery. Expert Opinion on Therapeutic Patents 2001; 11(9): 1395- 410.

13. Musial W, Kokol V, Voncina B. Deposition and release of chlorhexidine from non- onic and anionic polymer matrices. Chemical Papers 2010a; 64(3): $346-53$.
14. Musial W, Kokol V, Voncina B. Lidocaine hydrochloride preparations with ionic and non-ionic polymers assessed at standard and increased skin surface temperatures. Chemical Papers 2010b; 64(1): 84-90.

15. Diaf K, El bahri Z, Chafi N, Belarbi L, Mesli A. Ethylcellulose polycaprolactone, and eudragit matrices for controlled release of piroxicam from tablets and microspheres. Chemical Papers 2012; 66(8): 779-86.

16. Benoist JM, Montastruc JL, Giroud JP. Analgesiques coordinateurs, pharmacologie Clinique base de la therapeutique. Expansion Scientifique Française. $2^{\text {nd }}$ ed; 1988.

17. Higuchi T. Mechanism of sustained-action medication. Theoretical analysis of rate ofrelease of solid drugs dispersed in solid matrices. Journal Pharmaceutical Sciences 1963; 52(12): 1145-9.

18. Korsmeyer RW, Gurny R, Doelker E, Buri P, Peppas NA. Mechanisms ofsolute release from porous hydrophilic polymers. International Journal of Pharmaceutics 1983; 15(1): 25-35.

19. Hixson AW, Crowell JH. Dependence of reaction velocity upon surface and agitation. Ind. Eng. Chem. 1931; 23(10): 923-31.

20. Costa P, Lobo JMS. Modeling and comparison of dissolution profiles. Eur. J. Pharm. Sci. 2001; 13(2): 123-33.

21. Tanaka N, Imai K, Okimoto K, Ueda S, Tokunaga Y, Ohike A, Ibuki R, Higaki $\mathrm{K}$, et al. Development of novel sustained-release system, disintegrationcontrolled matrix tablet (DMCT) with solid dispersion granules of nilvadipine. J. Control. Release. 2005; 108(1): 386-96.

22. Champetier G, Monnerie L. Introduction à la Chimie Macromoléculaire Hermann Paris; 1975.

23. Parambil AM, Puttaiahgowda YM, Shankarappa P. Copolymerization of $\mathrm{N}$-vinyl pyrrolidone with methylmethacrylate by Ti(III)-DMG redox initiator. Turk. J. Chem. 2012; 36(3): 397-409.

24. Handbook of Chemistry and Physic, CRC Press. Florida. 60 th Ed; 1980.

25. Colombo P, Bettini R, Santi P, Peppas NA. Swellable matrices forcontrolled drug delivery: gel-layer behaviour, mechanisms and optimal performance. PSTT. 2000; 3(6): 198-204.

26. Kalam MA, Humayum M, et al, Release kinetics of modified pharmaceutical dosage forms: a review. Continental J. pharmaceutical Sciences 2007; 1: 30-5.

27. Siepmann J, Peppas NA. Modeling of drug release from delivery systems based on hydropropyl methylcellulose (HPMC). Adv. Drug Deliv. Rev. 2001; 48(2-3): 139-57. 